\title{
Brain-wide glymphatic enhancement and clearance in humans assessed with MRI
}

\author{
Geir Ringstad, ${ }^{1,2}$ Lars M. Valnes, ${ }^{3}$ Anders M. Dale, ${ }^{4,5,6}$ Are H. Pripp, ${ }^{7}$ Svein-Are S. Vatnehol, ${ }^{8}$ \\ Kyrre E. Emblem, ${ }^{9}$ Kent-Andre Mardal, ${ }^{3,10}$ and Per K. Eide ${ }^{2,11}$ \\ 'Division of Radiology and Nuclear Medicine, Department of Radiology, Oslo University Hospital - Rikshospitalet, Oslo, \\ Norway. ${ }^{2}$ Institute of Clinical Medicine, Faculty of Medicine, and ${ }^{3}$ Department of Mathematics, University of Oslo, Oslo, \\ Norway. ${ }^{4}$ Department of Neurosciences, ${ }^{5}$ Department of Radiology, and ${ }^{6}$ Multimodal Imaging Laboratory, UCSD, La Jolla, \\ California, USA. ${ }^{7}$ Oslo Centre of Biostatistics and Epidemiology, Research Support Services, Oslo University Hospital, Oslo, \\ Norway. ${ }^{8}$ The Intervention Centre, Oslo University Hospital - Rikshospitalet, Oslo, Norway. ${ }^{9}$ Department of Diagnostic \\ Physics, Oslo University Hospital, Oslo, Norway. ${ }^{10}$ Center for Biomedical Computing, Simula Research Laboratory, Lysaker, \\ Norway. ${ }^{11 D e p a r t m e n t ~ o f ~ N e u r o s u r g e r y, ~ O s l o ~ U n i v e r s i t y ~ H o s p i t a l ~-~ R i k s h o s p i t a l e t, ~ O s l o, ~ N o r w a y . ~}$
}

To what extent does the subarachnoid cerebrospinal fluid (CSF) compartment communicate directly with the extravascular compartment of human brain tissue? Interconnection between the subarachnoid CSF compartment and brain perivascular spaces is reported in some animal studies, but with controversy, and in vivo CSF tracer studies in humans are lacking. In the present work, we examined the distribution of a CSF tracer in the human brain by MRI over a prolonged time span. For this, we included a reference cohort, representing close to healthy individuals, and a cohort of patients with dementia and anticipated compromise of CSF circulation (idiopathic normal pressure hydrocephalus). The MRI contrast agent gadobutrol, which is confined to the extravascular brain compartment by the intact blood-brain barrier, was used as a CSF tracer. Standardized T1-weighted MRI scans were performed before and after intrathecal gadobutrol at defined time points, including at $\mathbf{2 4}$ hours, $\mathbf{4 8}$ hours, and $\mathbf{4}$ weeks. All MRI scans were aligned and brain regions were segmented using FreeSurfer, and changes in normalized T1 signals over time were quantified as percentage change from baseline. The study provides in vivo evidence of access to all human brain subregions of a substance administered intrathecally. Clearance of the tracer substance was delayed in the dementia cohort. These observations translate previous findings in animal studies into humans and open new prospects concerning intrathecal treatment regimens, extravascular contrast-enhanced MRI, and assessment of brain clearance function.

Conflict of interest: The authors have declared that no conflict of interest exists.

Submitted: April 6, 2018 Accepted: May 31, 2018 Published: July 12, 2018

\section{Reference information:} JCI Insight. 2018;3(13):e121537. https://doi.org/10.1172/jici. insight.121537.

\section{Introduction}

Knowledge about the access of substances administered in the subarachnoid space to human brain as whole could potentially make new treatments of brain disease. The blood-brain barrier (BBB) represents one of the largest obstacles to effective CNS drug delivery (1), and the compartment within blood vessels merely occupies less than $3 \%$ of the total brain volume (2). Thus, new therapeutic CNS drugs generally show lower success rates than those for non-CNS indications (3), while intrathecal treatment regimens have emerged with great promise $(4,5)$.

Previous animal studies have shown communication between the subarachnoid cerebrospinal fluid (CSF) space and perivascular compartments of the brain (6-10) and spinal cord (11). However, literature reporting human in vivo CSF tracer studies is lacking, and observations made in animals have not been translated into humans. Two recent human studies demonstrated brain parenchyma enhancement subsequent to subarachnoid (intrathecal) administration of a MRI contrast agent, but observations were limited to selected ROIs $(12,13)$. An MRI study of rats failed to demonstrate enhancement in deep brain white matter (14). Moreover, animal studies report diverging findings regarding the sites of perivascular brain influx and efflux and direction of perivascular flow $(7,15,16)$. Animal in vivo observations typically cover extremely limited fields of view, as when utilizing 2-photon microscopy (7).

Furthermore, mechanisms behind transport and clearance of substances within the brain interstitial space are controversial. For a long time, size-dependent diffusion was considered to explain 


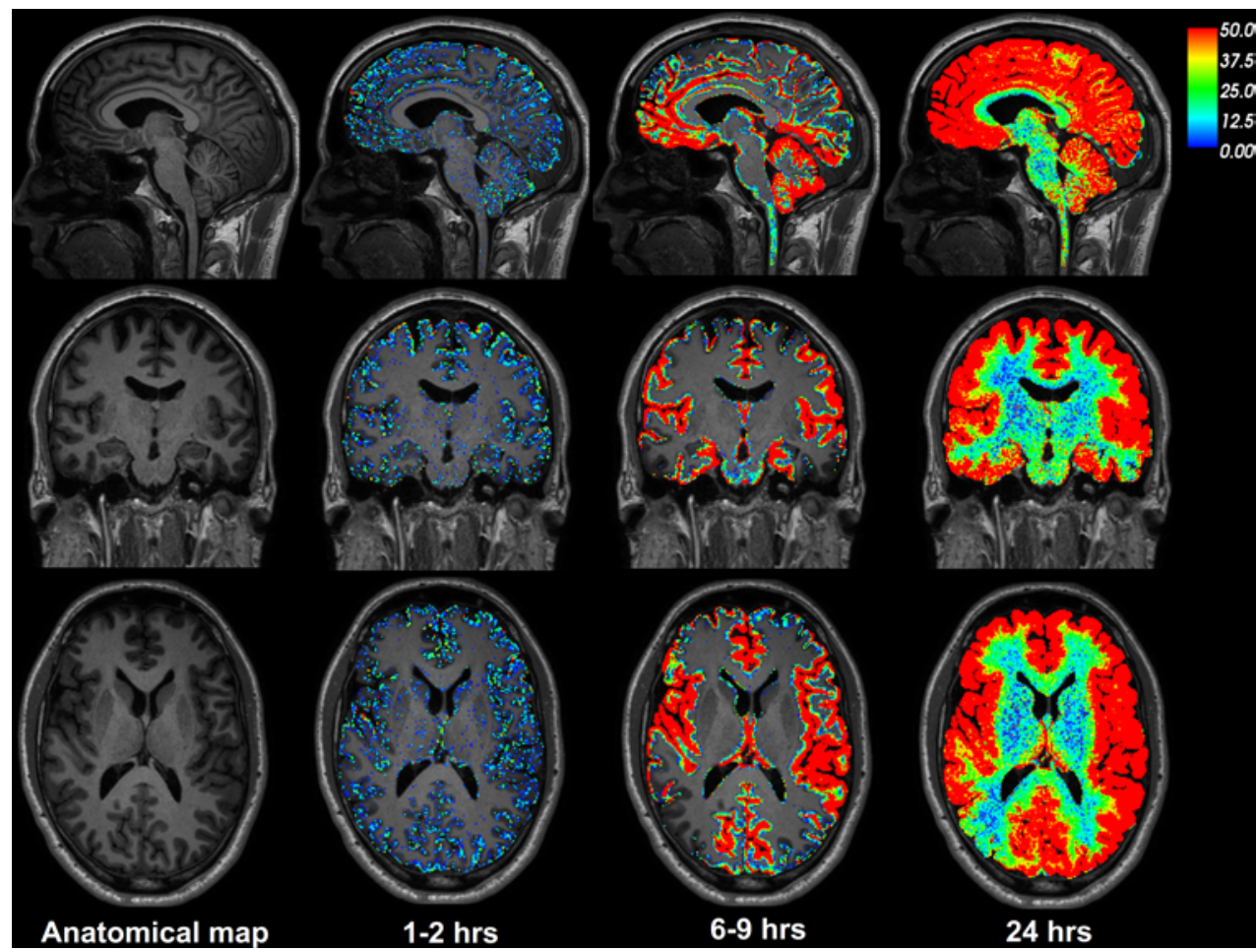

Figure 1. CSF tracer enrichment over time in one REF subject. The brain-wide enrichment of CSF tracer over time is shown for one REF individual. The percentage change in signal unit ratio is indicated by the color scale. CSF tracer enrichment occurred in a centripetal pattern at a rate exceeding the expected for pure diffusion, suggesting an important role of bulk flow for transport of substances through the brain. Tracer enrichment occurred primarily, and was most pronounced, in brain regions adjacent to large artery trunks at the surface (6-9 hours), underlining the important role of CSF pulsations for entry of substances into brain tissue perivascular spaces. Note the coronal section (middle row) demonstrating enhancement in the medial temporal lobe adjacent to the circle of Willis and posterior cerebral arteries, in the cingulum adjacent to the location of the anterior cerebral arteries in the anterior interhemispheric fissure, and around the Sylvian fissure, where the middle cerebral artery trunks reside.

interstitial movement of molecules (17). In 2012, a brain-wide pathway for convective transport of waste solutes from the brain was first described and denoted the glymphatic system (7). Net water and solute transport through the extracellular compartment from arterial to venous paravascular spaces was proposed to be dependent on aquaporin-4 (AQP4) water channels and mediated by arterial pulsations (18). Impaired glymphatic function has been suggested to be instrumental in a range of brain diseases; this has been illustrated most in Alzheimer's dementia $(7,19,20)$ but has also been shown to be relevant in posttraumatic encephalopathy (21), ageing (22), sleep (23, 24), depression (20), and exercise (25). However, the glymphatic concept has been challenged by several modeling studies that have opposed CSF pulsations as the explanation for net convective interstitial flow (26, 27). Moreover, a later animal study utilized a similar set of experiments as those bringing evidence for a glymphatic system and found, contrary to the previous observations, that interstitial flow could be explained by diffusion alone, independent of AQP4 status (9). Later, several independent groups provided evidence for an important role of AQP4 in glymphatic circulation (28).

To this end, we here show for what we believe to be the first time brain-wide CSF tracer enhancement and clearance in humans. For this, we administered an MRI contrast agent in the subarachnoid CSF compartment, followed by repeated MRI scans at 24 and 48 hours and after 4 weeks. In addition, we found delayed clearance of CSF tracer from the brain in a cohort of patients with dementia and expected CSF circulation failure (idiopathic normal pressure hydrocephalus [iNPH]).

\section{Results}

Brain-wide distribution of CSF tracer within brain parenchyma in reference subjects. The study includes 8 reference (REF) individuals who underwent MRI for tentative idiopathic intracranial hypotension due to 
Table 1. Percentage change in signal unit ratio at various time points after i.th. gadobutrol in REF individuals

\begin{tabular}{|c|c|c|c|c|c|c|c|c|}
\hline \multirow{3}{*}{$\begin{array}{l}\text { Anatomical region } \\
\text { [FreeSurfer] } \\
\text { CSF [24] }\end{array}$} & \multicolumn{7}{|c|}{ Time after i.th. gadobutrol } & \multirow{3}{*}{$\begin{array}{c}\text { Significance } \\
\quad<0.001\end{array}$} \\
\hline & $1.5-2$ hours & $2-4$ hours & 4-6 hours & $6-9$ hours & 24 hours & 48 hours & 4 weeks & \\
\hline & $16 \pm 20$ & $101 \pm 116$ & $354 \pm 309$ & $500 \pm 421$ & $157 \pm 164$ & 177 & $-8 \pm 17$ & \\
\hline $\begin{array}{l}\text { Cerebral cortex (gray matter) [1,000-1,035 } \\
\text { (left) }+2,000-2,035 \text { (right)] }\end{array}$ & $-3 \pm 7$ & $-1 \pm 15$ & $26 \pm 44$ & $44 \pm 50$ & $52 \pm 54$ & 61 & $-6 \pm 17$ & 0.018 \\
\hline Cerebral white matter $[2,41]$ & $-3 \pm 9$ & $-1 \pm 18$ & $26 \pm 40$ & $34 \pm 46$ & $27 \pm 33$ & 32 & $-10 \pm 17$ & 0.005 \\
\hline Basal ganglia $[11,12,13,50,51,52]$ & $-3 \pm 8$ & $-6 \pm 14$ & $1 \pm 25$ & $-4 \pm 14$ & $4 \pm 18$ & 32 & $-8 \pm 18$ & 0.014 \\
\hline Cerebellar cortex $[8,47]$ & $6 \pm 6$ & $12 \pm 27$ & $45 \pm 58$ & $67 \pm 80$ & $53 \pm 47$ & 90 & $-5 \pm 18$ & 0.003 \\
\hline Cerebellar white matter $[7,46]$ & $1 \pm 9$ & $-3 \pm 14$ & $3 \pm 23$ & $2 \pm 19$ & $15 \pm 23$ & 33 & $-7 \pm 18$ & 0.009 \\
\hline
\end{tabular}

Continuous variables are presented as mean \pm SD. Statistical differences were determined by a linear mixed model for repeated measurements. $n=8$. i.th., intrathecal. Numbers given in brackets refer to FreeSurfer predefined numbers for specific brain regions.

CSF leakage (Supplemental Table 1; supplemental material available online with this article; https://doi. org/10.1172/jci.insight.121537DS1). CSF leakage was diagnosed in 3 of 8 individuals; no CSF circulation disturbance was identified in the remaining 5 individuals. Even though the REF individuals had some complaints (Supplemental Table 1), we considered this cohort as close to healthy.

Figure 1 illustrates the distribution of CSF tracer in the brain parenchyma of one REF individual (see Supplemental Video 1). The tracer distributed centripetally from cortex to deeper brain regions. Supplemental Figure 1 shows the change in the T1 signal within the CSF space and brain parenchyma following intrathecal gadobutrol. Table 1 presents the percentage change in normalized T1 signal from before CSF tracer administration, including percentage change for cerebral cortex (gray matter), cerebral white matter, basal ganglia, thalamus, limbic structures (hippocampus, amygdala, nucleus accumbens, and entorhinal cortex), cerebellar cortex, and cerebellar white matter. The MRI signal increase was significant at all main locations (Table 1). Figure 2 shows the CSF contrast enrichment after 6-9 hours in coronal MRI sections of 8 REF individuals. As illustrated, the CSF tracer enrichment occurred in a centripetal pattern and primarily in brain regions adjacent to large artery trunks at the surface, i.e., the anterior, middle, and posterior cerebral arteries.

Figure 3 shows the percentage change in normalized T1 signal over time for selected brain subregions, including cerebral cortex (Figure 3A), cerebral white matter (Figure 3B), cerebellar cortex (Figure 3C), cerebellar white matter (Figure 3D), limbic structures (Figure 3E), basal ganglia (Figure 3F), corpus callosum (Figure 3G), and hippocampus (Figure 3H).

The REF individuals showed CSF tracer enrichment in close to all the brain regions studied, as further detailed in Supplemental Table 2.

Brain-wide distribution of CSF tracer in individuals with iNPH dementia. The study also includes 9 individuals with a subtype of dementia, denoted as iNPH (Supplemental Table 1). The iNPH patients were older than the REF subjects and presented with other symptoms (Supplemental Table 1). Figure 4 illustrates the color-coded enrichment of CSF tracer in brain parenchyma over time for one iNPH patient (see Supplemental Video 2). Enrichment in the periventricular white matter is a feature typical of $i \mathrm{NPH}$, due to ventricular tracer reflux and transependymal migration, as previously reported (13). Figure 5 shows the CSF contrast enrichment after 6-9 hours in coronal MRI sections of $8 \mathrm{iNPH}$ individuals. As for REF subjects, the CSF tracer enrichment occurred in a centripetal pattern and primarily in brain regions adjacent to large artery trunks at the surface. Table 2 presents the percentage of change in CSF tracer enrichment over time for selected brain regions of the iNPH subjects, including cerebral cortex (gray matter), cerebral white matter, basal ganglia, thalamus, and limbic structures as well as the cerebellar gray and white matter. CSF tracer enrichment was found in all these regions, and all subregions were defined in FreeSurfer (Supplemental Table 3).

Clearance of CSF tracer in REF subjects and iNPH patients. Our findings point to some important differences between REF subjects and individuals with iNPH dementia.

As illustrated in Figure 6, as compared with REF subjects, the CSF tracer enrichment in iNPH patients 


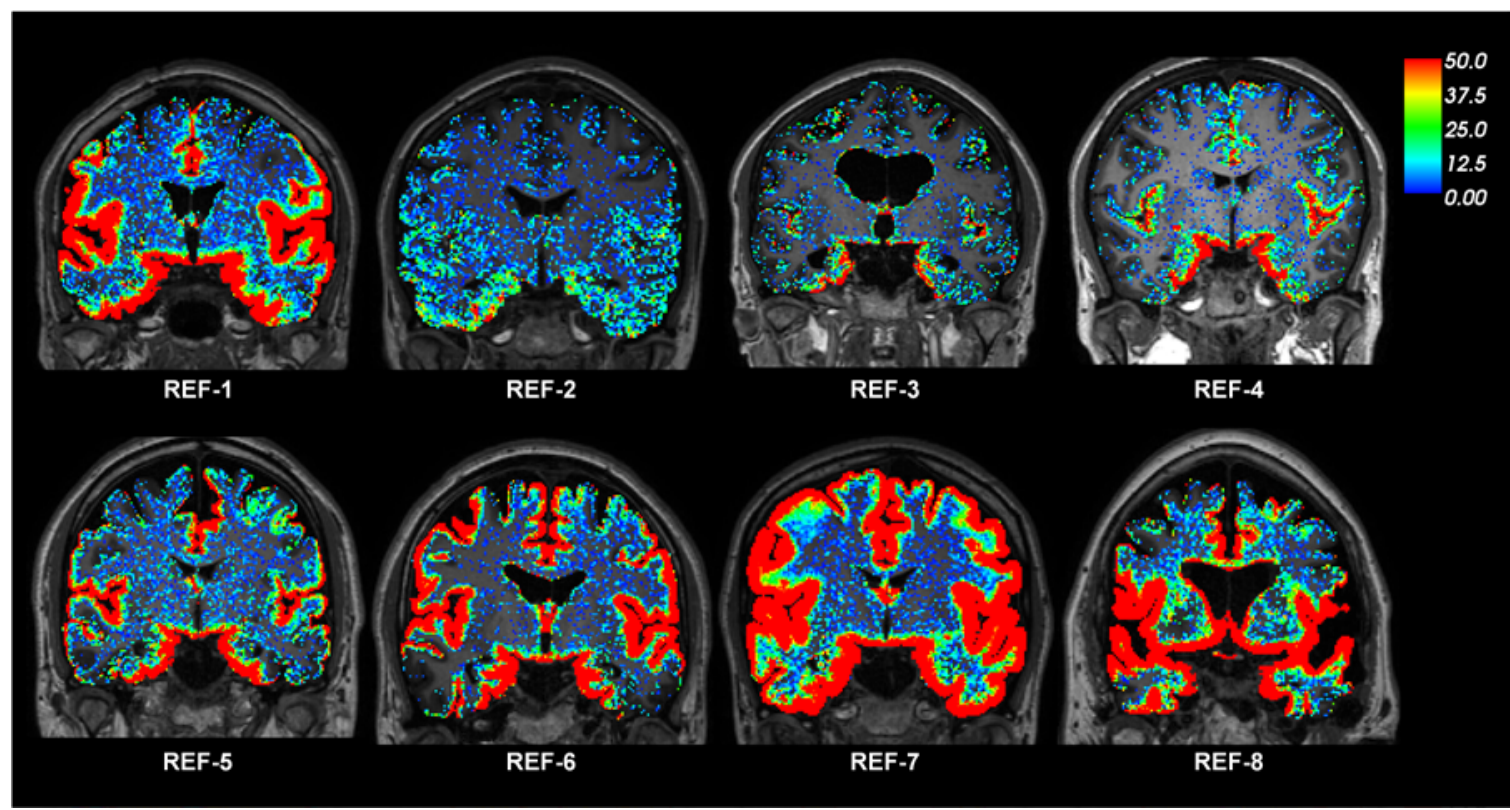

Figure 2. CSF tracer enrichment in REF subjects shows a centripetal pattern. The enrichment of CSF tracer after 6-9 hours is shown for 8 REF individuals. The percentage change in signal unit ratio is indicated by the color scale. The coronal sections illustrate that CSF tracer enrichment occurred in a centripetal pattern and primarily in brain regions adjacent to large artery trunks at the surface, i.e., the anterior, middle, and posterior cerebral arteries.

at 24 hours was significantly higher in cerebral white matter (Figure 6C), basal ganglia (Figure 6D), corpus callosum (Figure 6F), limbic system (Figure 6G), and hippocampus (Figure 6H), indicative of reduced tracer clearance. Supplemental Table 4 shows differences in percentages of change over time of normalized $\mathrm{T} 1$ signal for a wide range of brain regions.

CSF tracer enrichment after 4 weeks. We also compared the normalized T1 signal before and 4 weeks after intrathecal CSF tracer to look for signs of CSF tracer retention after 4 weeks. This was not found in any brain region examined for either REF or iNPH (Supplemental Table 5).

In a wide range of brain regions, the normalized T1 signal was consistently lower in iNPH individuals than in REF individuals before intrathecal gadobutrol and reached statistical significance for some of the regions (Supplemental Table 5). A lower T1 signal may be interpreted as indicative of higher brain parenchymal water content.

Correlation between CSF tracer enrichment and brain volume. The Pearson correlation coefficients between percentage change in signal unit ratios and tissue volume for white matter, gray matter, and CSF of lateral ventricles for REF and iNPH subjects are presented in Table 3. The correlation coefficients differ between $\mathrm{REF}$ and iNPH cohorts. In iNPH subjects, the volume of gray matter correlated negatively with CSF tracer enhancement in gray and white matter, i.e., with increasing volume the CSF tracer enhancement was lower.

\section{Discussion}

Brain-wide enhancement of CSF tracer in human brain. In this human CSF tracer study, repeated MR imaging provides in vivo evidence of a route for macromolecules administered in CSF from the subarachnoid compartment to the entire brain. Any substance with features similar to the studied tracer, gadobutrol, should therefore be expected to access all brain regions when administered intrathecally. We have previously demonstrated that an equally small amount of MRI contrast agent injected intrathecally at the lumbar level typically reaches the intracranial subarachnoid compartment in approximately 20 minutes, where it primarily distributes along large artery trunks at the brain surface (13). These observations were reproduced in the present work. Moreover, we reproduced the finding of primary tracer entry into the brain in tissue adjacent to major artery trunks, indicating an important role of CSF pulsations for macromolecular transport within brain tissue. Here, we have extended our assessment to cover all brain subregions, demonstrating tracer enhancement in locations even several centimeters deep to the brain surface and tracer bulk flow through the cerebral mantle. The CSF tracer enhancement within the brain occurred in a centripetal pattern, with some regional differences, and peak 
A

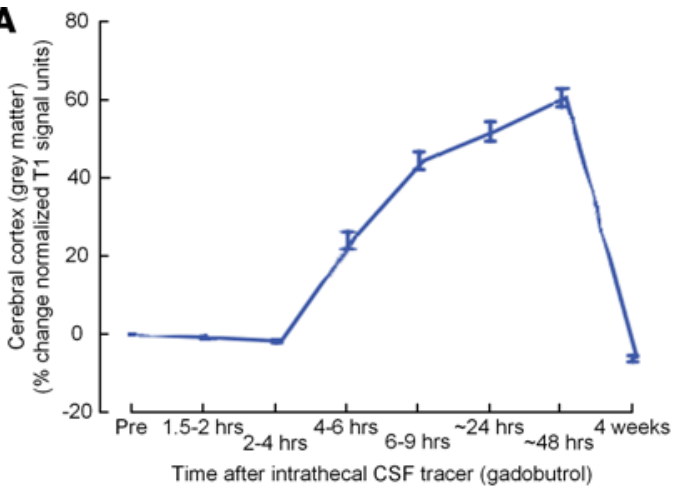

B

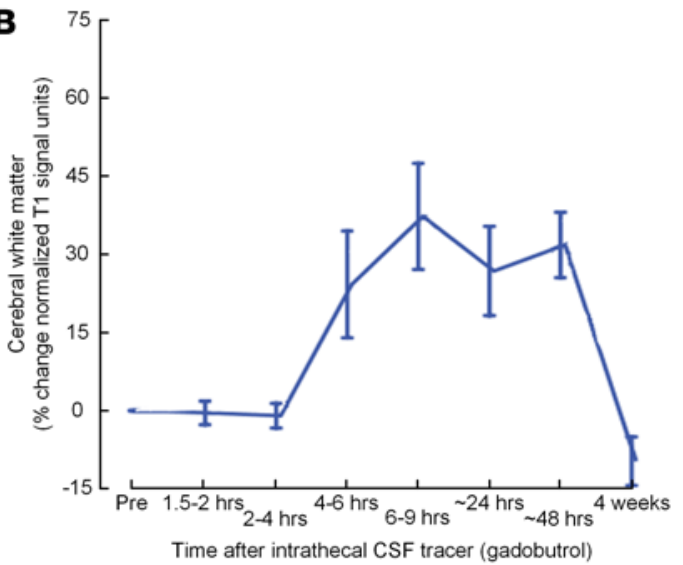

\section{C}

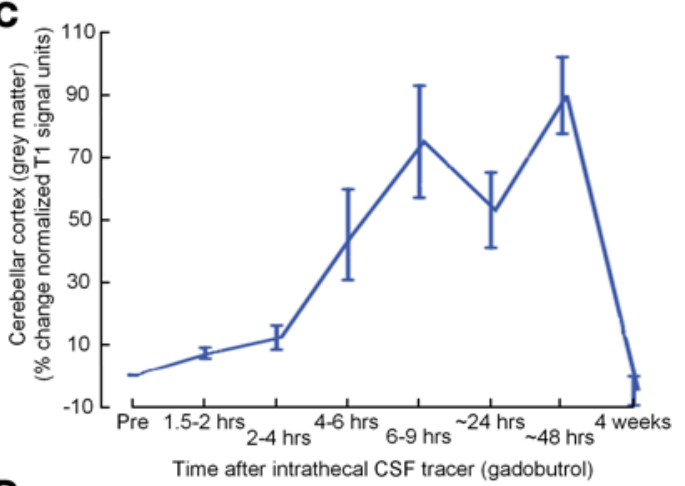

D

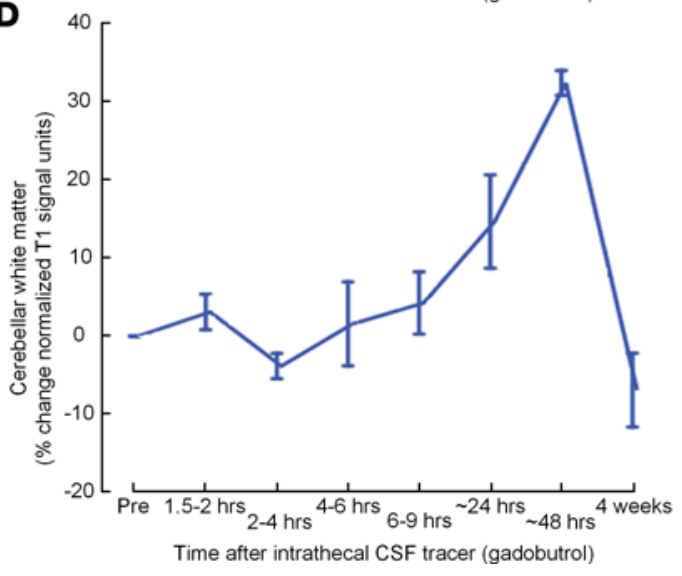

E

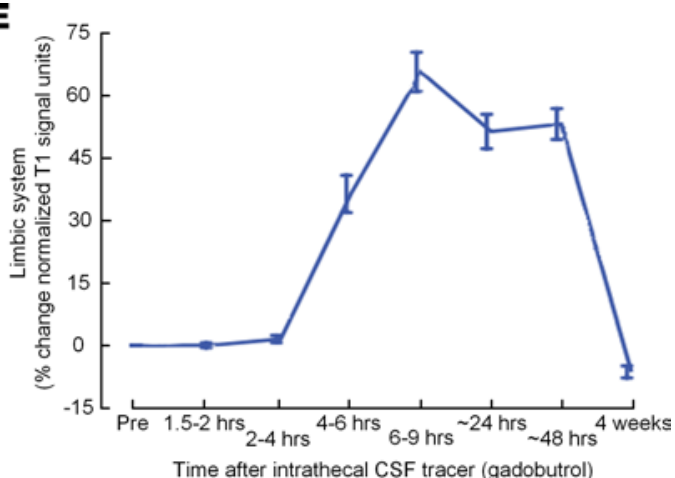

$\mathbf{F}$

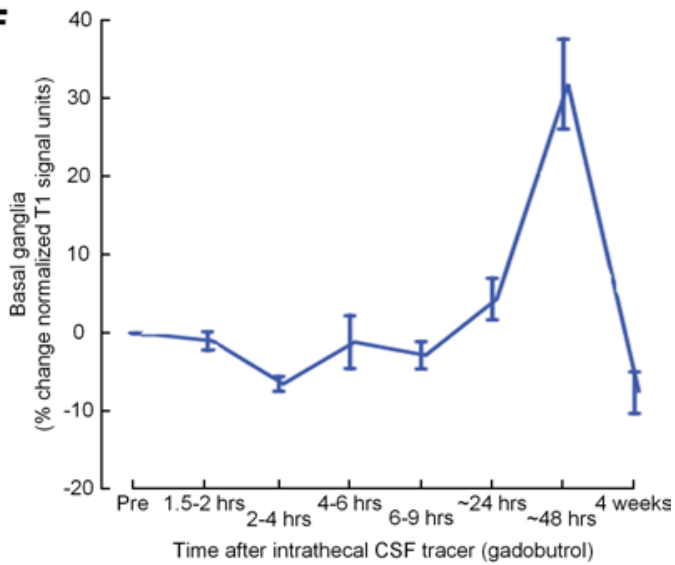

G

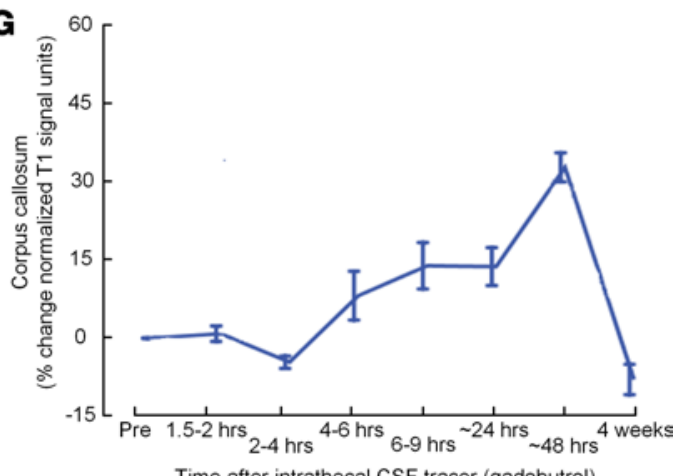

H

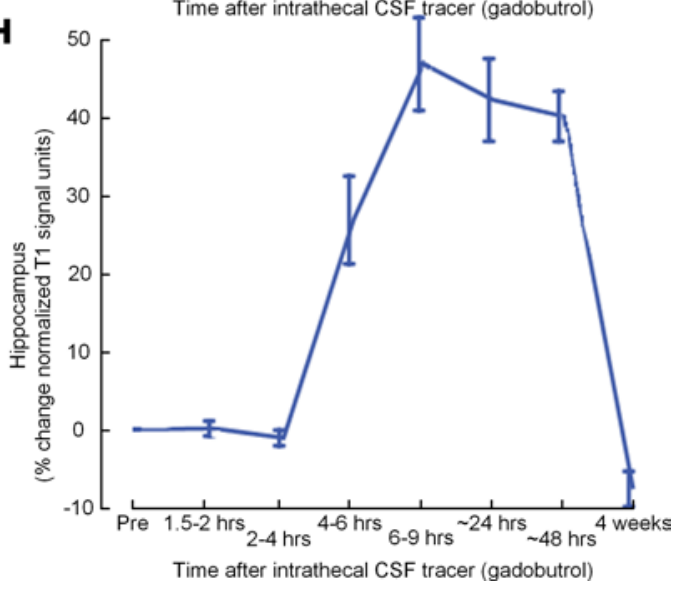

Figure 3. CSF tracer enrichment in some brain regions of the REF cohort. Trend plots of percentage change in signal unit ratio are presented for main regions within the supratentorial and infratentorial compartments, including (A) cerebral cortex (gray matter), (B) cerebral white matter, (C) cerebellar cortex, (D) cerebellar white matter, (E) limbic system, (F) basal ganglia, (G) corpus callosum, and (H) hippocampus. Trend plots are presented with mean \pm standard error (SE). $n=8$. 


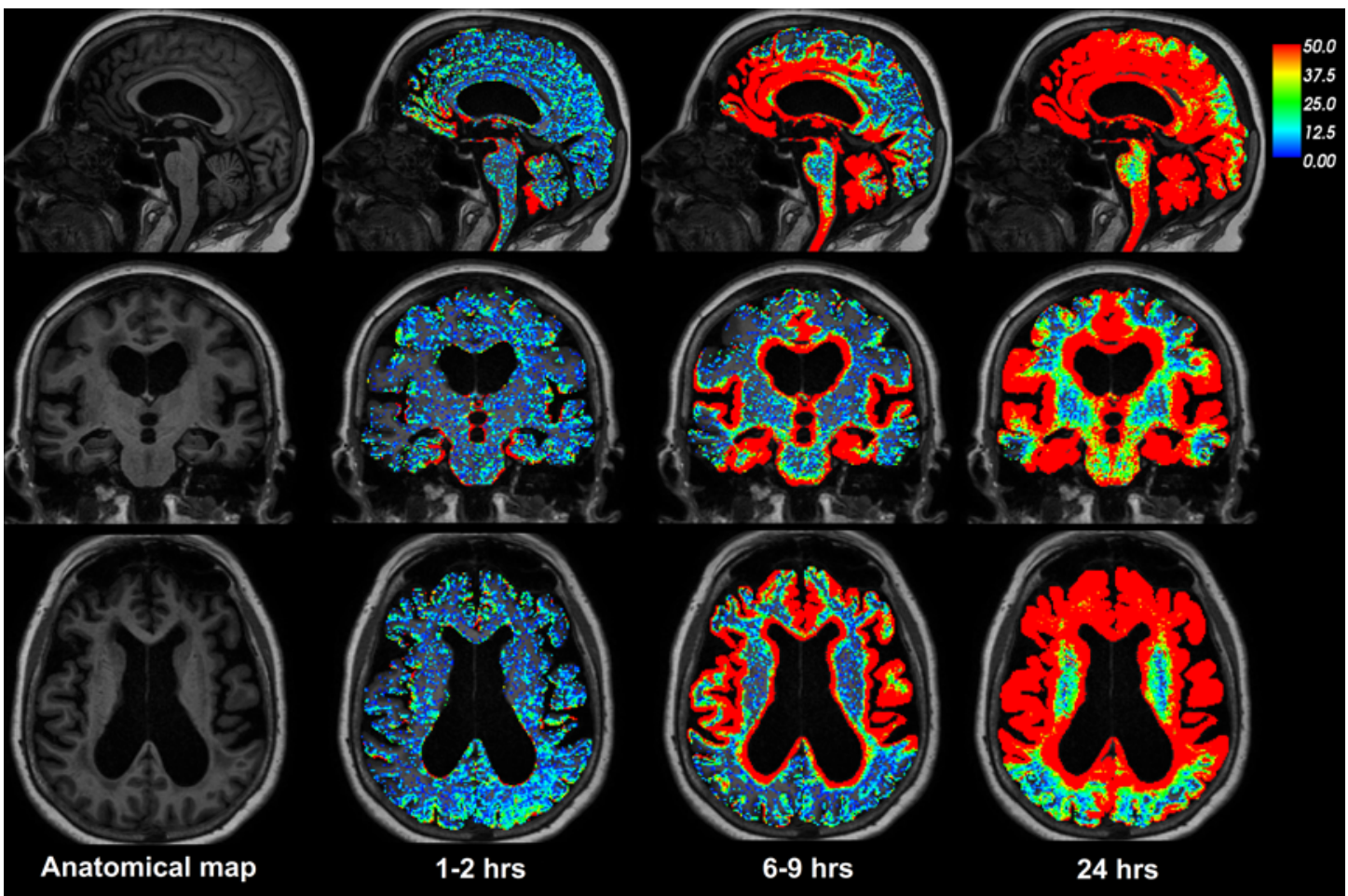

Figure 4. CSF tracer enrichment over time in one iNPH subject. The brain-wide enrichment of CSF tracer over time is shown in one iNPH individual. The percentage change in signal unit ratio is indicated by the color scale. The centripetal pattern of enrichment is comparable with that in REF individuals. In $\mathrm{iNPH}$, there is also enrichment of periventricular white matter due to ventricular reflux of CSF tracer substance (ventricular CSF space is subtracted). While clearance of tracer was delayed in iNPH, enrichment occurred with at least the same rate as in REF. This may be attributed to enlarged perivascular spaces in $\mathrm{iNPH}$, where neurodegeneration and vascular disease typically are important associated features. Regions with the initial, and most, tracer enrichment are nearby the course of large artery trunks at the brain surface (as further outlined in the legend to Figure 1), indicating the important role of CSF pulsations for brain tracer enrichment. These regions (entorhinal, hippocampus, insula, cingulum, etc.) also correspond to areas that traditionally are included in the limbic system ("limbic enhancement") and also are areas typically susceptible to consecutive tau propagation in Alzheimer's disease. This should be noted, as reduced clearance of CSF tracer through the glymphatic system has been proposed as instrumental in the pathogenesis of neurodegenerative disease.

enhancement typically occurred at 24 hours after tracer injection. Thereafter, the level of tracer in parenchyma declined, and tracer had been cleared from the brain after 4 weeks.

Mechanisms behind brain cortex tracer enrichment. The hydrodynamic diameter of gadobutrol has, to our knowledge, not been estimated previously. However, prediction of hydraulic diameter based on molecular weight (MW) is feasible (29). With a MW of 3,000 Da, dextran 3 is comparable to the highly hydrophilic molecule gadobutrol with respect to $\mathrm{MW}(\mathrm{MW}=604 \mathrm{Da})$, and the hydrodynamic diameter of dextran 3 has been experimentally calculated as $26 \AA$ or $2.6 \mathrm{~nm}$ (30). Hydraulic diameter of gadobutrol is therefore not expected to exceed this, and further distribution within the brain cortex interstitial compartment should accordingly be expected to be predominantly driven by diffusive flux (1). Average in vivo width of extracellular space has been reported to be between 38 and $64 \mathrm{~nm}$ (31). In ref. 17, the timescale of the relative diffusive tracer distribution (C) is estimated as follows: $C=\operatorname{erfc}(x /(2 \times \sqrt{ }(D \times t))$. Using this formula, assuming a diffusivity of $D=12 \times 10^{-7} \mathrm{~cm}^{2} / \mathrm{s}$, with $x$ and $t$ corresponding to the length in $\mathrm{cm}$ and time in seconds, respectively, a $50 \%$ saturation of the extracellular space can be estimated to occur at around 55 hours. The assumptions underlying this formula are, however, that the length scale is such that the cortex can be considered flat and the tracer distribution uniform. These assumptions are not valid in our case, but it seems unlikely that diffusion alone explains the brain-wide distribution. Further, loss of the molecule across the $\mathrm{BBB}$, uptake into cells, or binding to receptors all may modify diffusion (17).

It has been proposed that the perivascular compartment of human cortex is merely a potential space for movement of solutes (32-36), a view that recently was maintained based on observations made in fixed tissue specimens (37). In contrast, others have reported that the intrinsic hydraulic permeability of the human perivascular space is far greater than the width of the cortical extracellular space (1). The width of the perivascular space is suggested to be at least 2 orders of magnitude greater than neocortical extracellular 
Table 2. Percentage change in signal unit ratio at various time points after i.th. gadobutrol in iNPH individuals

\begin{tabular}{|c|c|c|c|c|c|c|c|c|}
\hline \multirow{2}{*}{$\begin{array}{l}\text { Anatomical region } \\
\text { [FreeSurfer] }\end{array}$} & \multicolumn{7}{|c|}{ Time after i.th. gadobutrol } & \multirow[t]{2}{*}{ Significance } \\
\hline & $1.5-2$ hours & 2-4 hours & 4-6 hours & $6-9$ hours & 24 hours & 48 hours & 4 weeks & \\
\hline CSF [24] & $23 \pm 29$ & $94 \pm 80$ & $448 \pm 346$ & $755 \pm 323$ & $719 \pm 363$ & $256 \pm 304$ & $2 \pm 16$ & $<0.001$ \\
\hline $\begin{array}{l}\text { Cerebral cortex (gray matter) }[1,000- \\
1,035 \text { (left) }+2,000-2,035 \text { (right) }]\end{array}$ & $4 \pm 20$ & $3 \pm 14$ & $23 \pm 25$ & $37 \pm 30$ & $91 \pm 46$ & $66 \pm 57$ & $5 \pm 17$ & $<0.001$ \\
\hline Cerebral white matter $[2,41]$ & $3 \pm 20$ & $3 \pm 16$ & $41 \pm 39$ & $53 \pm 43$ & $66 \pm 38$ & $34 \pm 39$ & $2 \pm 16$ & $<0.001$ \\
\hline Basal ganglia $[11,12,13,50,51,52]$ & $4 \pm 21$ & $0 \pm 15$ & $4 \pm 20$ & $5 \pm 27$ & $27 \pm 25$ & $22 \pm 25$ & $2 \pm 17$ & $<0.001$ \\
\hline Cerebellar cortex $[8,47]$ & $7 \pm 18$ & $9 \pm 16$ & $30 \pm 28$ & $53 \pm 35$ & $96 \pm 38$ & $52 \pm 43$ & $9 \pm 16$ & $<0.001$ \\
\hline Cerebellar white matter $[7,46]$ & $4 \pm 19$ & $1 \pm 15$ & $6 \pm 18$ & $4 \pm 16$ & $27 \pm 24$ & $19 \pm 17$ & $4 \pm 16$ & $<0.001$ \\
\hline
\end{tabular}

Continuous variables given as mean \pm SD. Statistical differences determined by a linear mixed model for repeated measurements. $n=9$. i.th., intrathecal. Numbers given in brackets refer to FreeSurfer predefined numbers for specific brain regions.

space width, and intrinsic hydraulic permeability of the perivascular space is suggested to be at least 10,000 fold higher than the extracellular space of the neuropil. It is therefore reasonable to assume that the cortical tracer enhancement in our human cohorts derives from the same mechanism as that reported in animal studies, where perivascular CSF tracer distribution in animal cortex is consistently demonstrated (6-8). Human cortical enhancement was, however, a much more durable process (days) than that of animals (hours) (14). The role of vascular pulsations being mediated to CSF for enrichment of perivascular spaces is underlined by the rich and early enhancement in parenchyma adjacent to large arterial vessels at the brain surface (Figures 1, 2, 4, and 5). Here, diffusion likely adds to advective pulsatile to-and-fro flow, i.e., dispersion (38). Dispersion by itself does not require net, convective flow. Based on animal studies (6, 7, 39), we expected further transport of tracer into the interstitial space to occur at the neurovascular interface through astrocytic inter-endfeet gaps, which allow for passage of solutes of up to 20-nm hydraulic diameter (38).

Tracer enrichment in deep brain structures. CSF tracer also propagated in brain tissue deeper than what can be attributed to perivascular spaces connected to the brain surface only. The present in vivo observations of contrast enhancement in deep white matter extends evidence from a similarly designed MRI study of rats, where lack of enhancement in deep portions of the brain white matter was attributed to concentrations of contrast agent probably being too small to be detected. (14). Image resolution of the MRI scan was 1 $\mathrm{mm}$, while the sizes of paravascular and interstitial spaces are in the order of $\mu \mathrm{m}$. A detailed assessment of molecular motion at a microscopic level was therefore beyond reach in this study.

Previous animal studies have indicated that axon tracts, in addition to perivascular spaces, can also act as preferential, low-resistance pathways for solutes through the brain (40). White matter has been identified as a major site for convective flow under normal conditions (41). In a recent study, interconnections between perivascular compartments of arterioles and veins, allowing small and large molecules to bypass the interstitial space, were demonstrated (42). Diffusion alone has also been proven inefficient at distances larger than a few millimeters, as in the human brain (43). Furthermore, tracers of different MW are cleared at similar speeds in the brain, which is not consistent with diffusion, but rather convective flow (44). Our study observations, with enrichment of CSF tracer over a range of several centimeters within 24 hours, may suggest that convective forces and bulk flow in general are more important for brain-wide tracer distribution than diffusion alone. Bulk flow has previously proven difficult to quantify (17) but is considered most likely to be restricted to the perivascular spaces (40). One study estimated the velocity of white matter bulk flow toward the ventricles as $10.5 \mu \mathrm{m} / \mathrm{min}$ (approximately $15 \mathrm{~mm}$ per day) (41). In our REF subjects, enhancement in regions several $\mathrm{cm}$ deep to the brain surface was found at 24 hours (Figures 1-3; Table 1; and Supplemental Table 2), which by far exceeds previous estimates for bulk flow. Parenchymal migration of a substance over a distance much farther than previously shown in both animals and humans also has important implications for the potential of neurotransmitters at a synapse to reach receptors outside the immediate postsynaptic site. Such extrasynaptic volume transmission has not been explicitly identified before and has therefore remained an elusive concept with respect to intercellular communication in the CNS (17). 


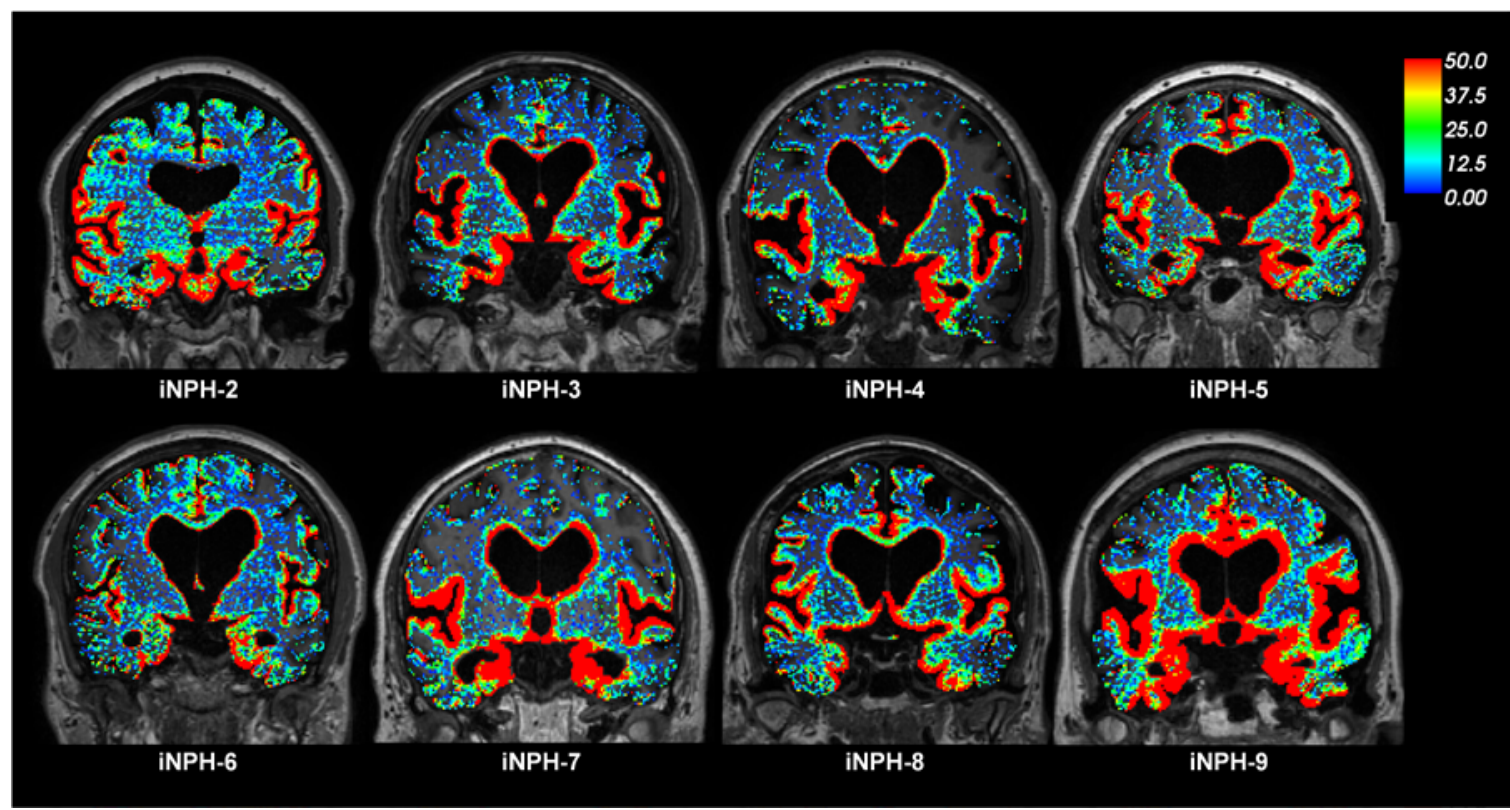

Figure 5. CSF tracer enrichment in iNPH subjects shows a centripetal pattern. The enrichment of CSF tracer after 6-9 hours is shown for 8 iNPH individuals (MRI after 6-9 hours was lacking in one iNPH individual). The percentage change in signal unit ratio is indicated by the color scale. The coronal sections illustrate that CSF tracer enrichment occurred in a centripetal pattern and primarily in brain regions adjacent to large artery trunks at the surface, i.e., the anterior, middle, and posterior cerebral arteries. Periventricular tracer enhancement due to reflux of tracer into the ventricular system is a typical feature of iNPH (13).

Effect of sleep. One mechanism behind the observed high bulk flow rate could be the effect of sleep. Natural sleep in animal studies has been shown to associate with increased size of the interstitial space, facilitating increased convective exchange of CSF with interstitial fluid and clearance of macromolecules (23). While we notice that the observed tracer enhancement in brain generally peaked after the first night (at 24 hours), which is remarkable, we do not have a sufficient number of MRIs in the time interval from 6-9 hours to 48 hours to conclude the exact time point of when peak enhancement truly occurred. It should also be noted that tracer enrichment of cortex and white matter was in progress before onset of sleep (Figures 1-3; Table 1; and Supplemental Table 2). Peak enhancement occurring at 24 hours differs significantly from that found in studies of rats, which typically was at 1-3 hours $(14,45)$.

Tracer clearance routes from CSF and brain parenchyma. The centripetal pattern of brain enhancement seems to contradict studies in which intraparenchymal tracers have been injected and described as leaving the brain centrifugally along the walls of arteries $(15,46)$. The findings made in these previous tracer studies have led to the hypothesis that pathological aggregates of amyloid- $\beta$ in walls of cortical arteries are indicative of $\mathrm{AD}$ pathogenesis. Widespread penetration of substances from CSF into brain parenchyma along perivascular spaces of arteries is, however, well supported by animal studies with a similar experimental setup to ours $(1,47-49)$. While our present study demonstrated declining levels of the tracer molecule after 24 hours, and no remains at 4 weeks, the routes for tracer clearance are still elusive. Uptake into arachnoid villi, as proposed more than one hundred years ago, seems to be of minor importance in humans, as reviewed by Brinker et al. (50) and as indicated in our recent study (13). In 2015, lymphatic vessels draining from CSF to neck lymph nodes were for the first time detected in walls of dural venous sinuses $(51,52)$. The full significance of these lymphatic drainage pathways has yet to be shown, but we have found that neck lymph node and brain tracer enhancement coincided in time, indicating this drainage is from the brain rather than directly from CSF (53). Should lymphatic drainage be directly from CSF, as suggested in a recent animal study (54), this would then occur much slower in humans than reported in animals. Our present findings of peak tracer enhancement in CSF at 6-9 hours, followed by a decline before peak enhancement in brain occurs (24 hours; Figure 3 and Figure 6), contradicts that the levels of tracer in $\mathrm{CSF}$ and parenchyma are merely balanced by diffusion between these compartments. Because CSF tracer clearance along veins is suggested in several studies $(6-8,39)$, we found it reasonable to hypothesize that perivenous compartments and lymphatic vessels are interconnected. 
A

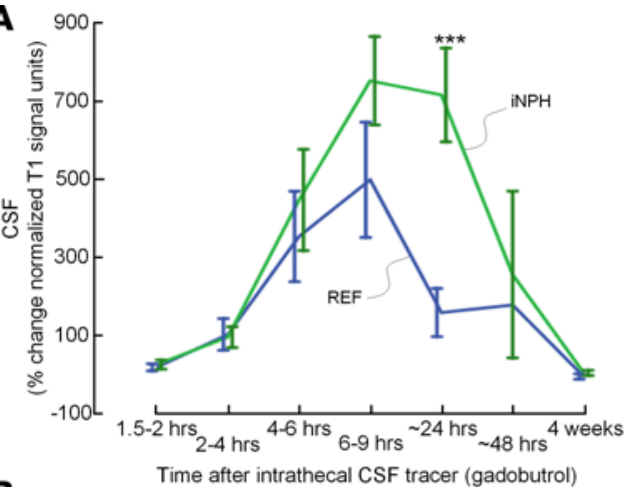

B

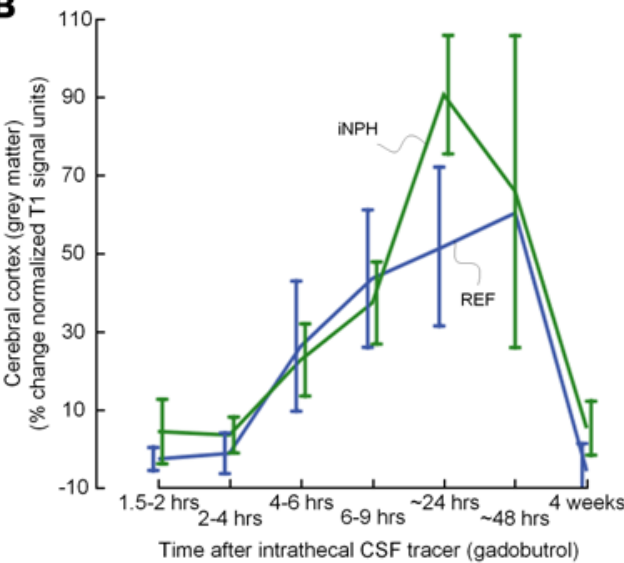

C

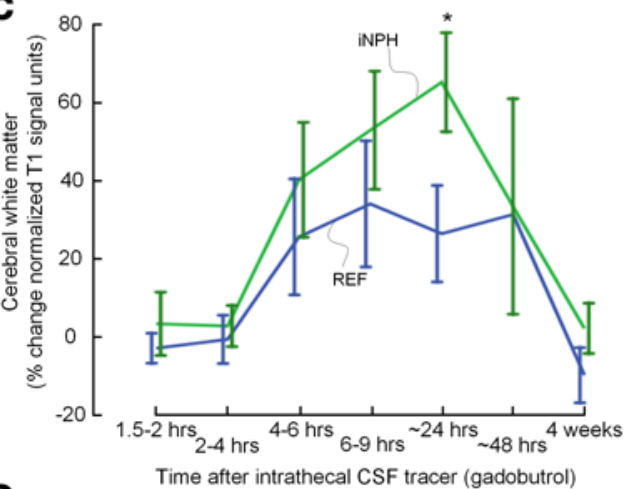

D

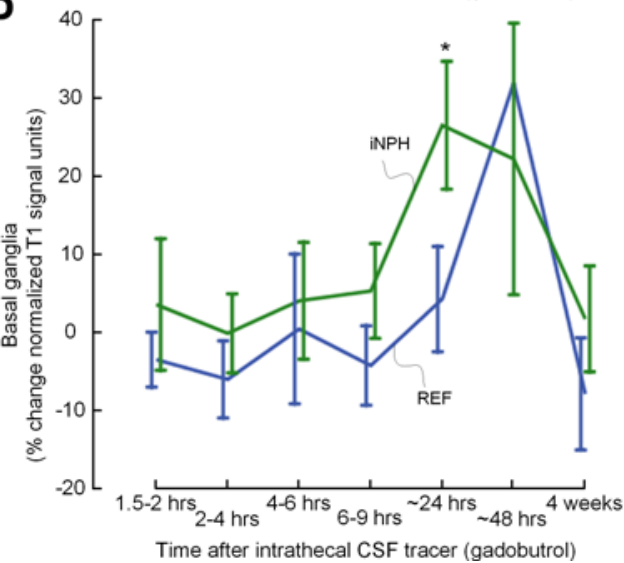

E

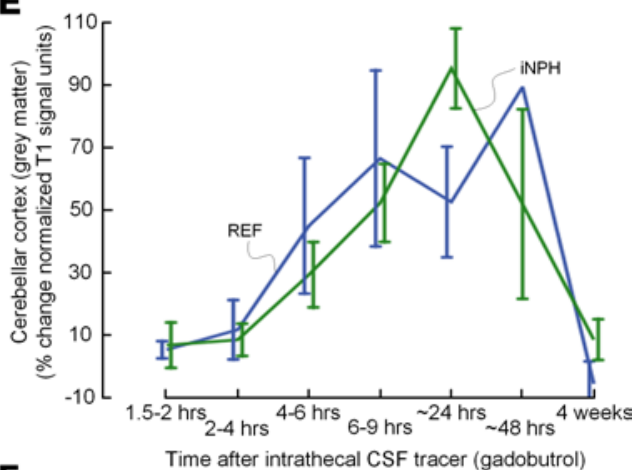

$\mathbf{F}$

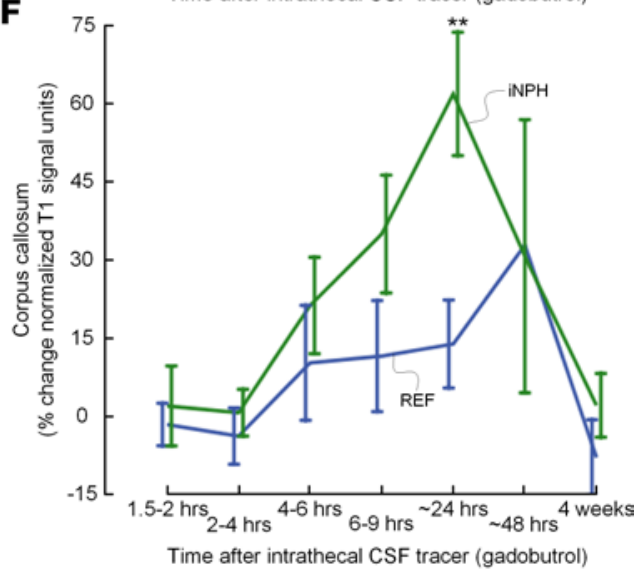

G

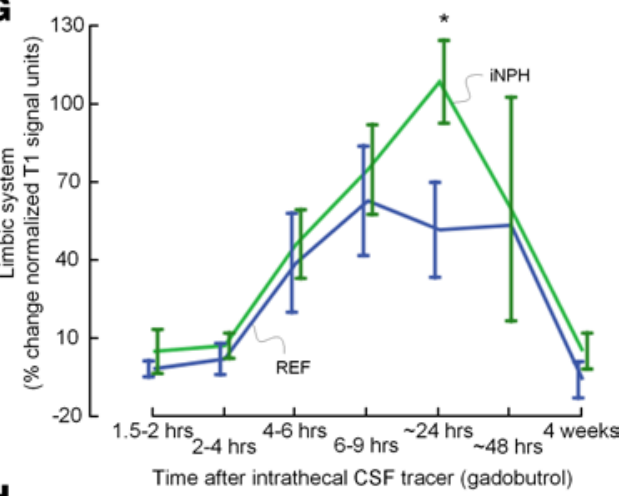

H

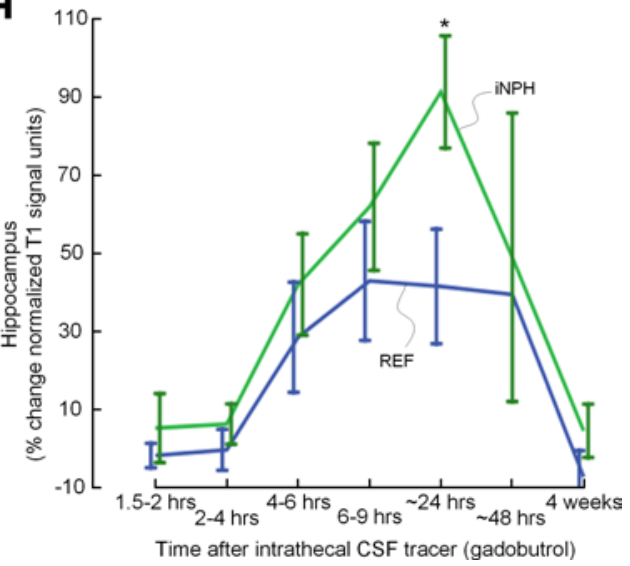

Figure 6. Comparison of CSF tracer enrichment between REF and iNPH subjects within selected brain regions. Trend plots of percentage change in signal unit ratio are presented for different regions within the supratentorial and infratentorial compartments, including (A) CSF, (B) cerebral cortex (gray matter), (C) cerebral white matter, (D) basal ganglia, (E) cerebellar cortex, (F) corpus callosum, (C) limbic system, and (H) hippocampus. Significant differences between REF (blue line) and iNPH (green line) groups were determined by linear mixed models for repeated measurements. ${ }^{*} P<0.05,{ }^{* *} P<0.01$, ${ }^{* *} P<0.001$. Trend plots are presented with mean \pm standard error (SE). $n=8, \mathrm{REF} ; n=9$, iNPH. 
Table 3. Correlation between CSF tracer enrichment at 24 hours and volume of the specific brain region

\begin{tabular}{|c|c|c|c|}
\hline & $\begin{array}{l}\text { CSF contrast enhancement in gray } \\
\text { matter (\%) }\end{array}$ & $\begin{array}{l}\text { CSF contrast enhancement in white } \\
\text { matter (\%) }\end{array}$ & $\begin{array}{l}\text { CSF contrast enhancement in lateral } \\
\text { ventricles (\%) }\end{array}$ \\
\hline \multicolumn{4}{|l|}{ REF patients } \\
\hline Volume of gray matter $\left(\mathrm{mm}^{3}\right)$ & $\mathrm{R}=0.11(\mathrm{NS})$ & $\mathrm{R}=0.08$ (NS) & $\mathrm{R}=-0.60(\mathrm{NS})$ \\
\hline Volume of white matter $\left(\mathrm{mm}^{3}\right)$ & $\mathrm{R}=0.21(\mathrm{NS})$ & $\mathrm{R}=0.25$ (NS) & $\mathrm{R}=-0.31(\mathrm{NS})$ \\
\hline Volume of lateral ventricles $\left(\mathrm{mm}^{3}\right)$ & $R=-0.037(N S)$ & $\mathrm{R}=-0.36(\mathrm{NS})$ & $\mathrm{R}=0.92(P=0.003)$ \\
\hline \multicolumn{4}{|l|}{ iNPH patients } \\
\hline Volume of gray matter $\left(\mathrm{mm}^{3}\right)$ & $\mathrm{R}=-0.78(P=0.012)$ & $\mathrm{R}=-0.81(P=0.008)$ & $\mathrm{R}=-0.13(\mathrm{NS})$ \\
\hline Volume of white matter $\left(\mathrm{mm}^{3}\right)$ & $\mathrm{R}=-0.33(\mathrm{NS})$ & $\mathrm{R}=-0.46(\mathrm{NS})$ & $\mathrm{R}=-0.33(\mathrm{NS})$ \\
\hline Volume of lateral ventricles $\left(\mathrm{mm}^{3}\right)$ & $\mathrm{R}=-0.37(\mathrm{NS})$ & $\mathrm{R}=-0.21(\mathrm{NS})$ & $\mathrm{R}=0.41(\mathrm{NS})$ \\
\hline \multicolumn{4}{|c|}{ Pearson correlation coefficients, with significance level. Gray matter corresponds to FreeSurfer regions 1,000-2,999, white matter regions correspond to } \\
\hline \multicolumn{4}{|c|}{$\begin{array}{l}\text { FreeSurfer regions 3,000-5,002, and lateral ventricles correspond to FreeSurfer regions } 4 \text { and } 43 \text {. The CSF contrast enrichment refers to percentage change } \\
\text { in signal unit ratios after } 24 \text { hours as compared with baseline. }\end{array}$} \\
\hline
\end{tabular}

Therapeutic and diagnostic implications of brain-wide tracer enhancement. The potential for administrating drugs via the intrathecal route has been considered to be limited by some (55). Compared with the intravenous route, intrathecal delivery of drugs in treatment of neurological disease is still rare, and few drugs have been approved for this use. Because many neurological conditions primarily are diseases of the interstitial space, and do not cause breakdown of the BBB, brain-wide access to the extravascular CNS compartment via intrathecal delivery may show to have many benefits. An already established treatment regime is that of intrathecal monoclonal antibodies against multiple sclerosis (56). Other medications approved by the US Food and Drug Administration for intrathecal use include morphine, ziconotide, and baclofen (57). Studies of intrathecal treatment for infantile-onset spinal muscular atrophy are emerging with promising results (4), and intrathecal delivery of recombinant enzyme to patients with mucopolysaccharidosis type II (Hunter syndrome) has been proposed (47).

Knowledge of what extent an intrathecal substance with certain size and properties can distribute throughout the entire human brain may help pave the way for new treatment options. It is likely that monoclonal antibodies and viral vectors for gene therapy could provide new prospects for treatment of neurodegenerative disorders, such as Alzheimer's and Parkinson's disease $(1,58)$. Furthermore, neoplastic and inflammatory processes that primarily occur in the extravascular compartment could more readily be targeted for diagnostic purposes. For the latter, contrast-enhanced imaging of the extravascular space may increase sensitivity for detection of extravascular inflammatory (59) and neoplastic diseases (60), which probably are most likely missed with conventional MRI techniques. With contrast-enhanced imaging, disease-targeting substances may be conjugated with contrast agents to mark disease and monitor treatment.

There are to date no gadolinium-based MRI contrast agents approved for intrathecal use. In the present study, gadobutrol was used after special permission granted from the National Medicine Agency of Norway. Gadolinium accumulation in the brain after multiple intravenous administrations has been a major concern since it was first reported in 2014 (61). However, there have been no reports demonstrating brain toxicity (62); the US Food and Drug Administration and American College of Radiology have declared that there is no evidence to date that gadolinium accumulation in the brain is harmful and that there is no need to restrict its intravenous usage. In the present study, where intrathecal administration of an MRI contrast agent was utilized, no serious adverse events were noted among our study subjects, and at the 4-week MRI follow-up, no evidence of remaining gadobutrol within brain parenchyma or CSF was found (Supplemental Table 5). Interestingly, gadolinium deposits in brain tissue have recently been attributed to leakage from blood into CSF through the choroid plexus and entrance into the brain from the surface along perivascular spaces (62). In rats, gadolinium concentration has been shown to be higher in CSF than blood 4.5 hours after intravenous distribution and highest in brain after 24 hours (63). In principle, an intravenous dose could therefore potentially expose the brain perivascular compartment to a larger amount of contrast agent than a very small intrathecal dose, as used in this study. Macrocyclic contrast agents (including gadobutrol) are now generally preferred above linear agents, which are chemically less stable compounds and more susceptible to release into tissue $(64,65)$. 
Reduced clearance of CSF tracer in iNPH. The other main finding of this study is the demonstration of reduced clearance of gadobutrol used as CSF tracer in a cohort of patients with dementia. Clearance of MRI contrast agents, such as gadobutrol, has previously been proposed to potentially resemble clearance of brain macromolecules with similar properties (14), including amyloid- $\beta$ (7) and tau $(21,66)$. These proteins are found pathologically accumulated as insoluble amyloid- $\beta$ and as component of neurofibrillary tangles, respectively, in brains of patients with Alzheimer's disease (67). There is large overlap of typical findings in brain specimens of patients with Alzheimer's disease and iNPH (68). We have recently demonstrated volume loss and reduced tracer clearance in a iNPH dementia cohort at the entorhinal area (69), where the first signs of neurodegeneration appear in Alzheimer's disease (70). The apparent important role of artery pulsations in parenchymal tracer propagation compares well with findings of vascular comorbidity (71) and reduced subarachnoid space propagation of CSF tracer in iNPH (13). Furthermore, brain subregions being closest to large artery trunks at the brain surface are regions traditionally considered as part of the limbic system (entorhinal cortex, hypothalamus, insula, amygdala, cingulum, etc.), representing the phylogenetically oldest part of the cerebral cortex (allocortex). On the MRIs, these regions enhanced earlier and enhancement was richer compared with other brain regions (Figures 1,2, 4 and 5; Table 2; Supplemental Table 3). We find it compelling how this pattern of time-progressive "limbic enhancement" resembles the pattern of neurofibrillary tangle advancement in evolving Alzheimer's disease (72), which to date is incompletely understood. A contributing cause to disease evolvement might therefore be that brain regions being most dependent on CSF pulsations for clearance of brain macromolecules are also the ones most susceptible to disease when pulsations are restricted or when paravascular pathways are blocked by any cause. To what extent restricted CSF pulsations and restricted paravascular flow may be instrumental in the pathogenesis behind Alzheimer's disease, iNPH, and other brain proteinopathies should be subject to future studies.

In the present study, the 24-hour time point showed the best discrimination between patients and REF individuals. Assessment of cerebral clearance with MRI by absolute quantities is, however, not possible at this level, as normalized T1 signal cannot be assumed to be strictly proportional with amount of contrast agent in each image voxel. Another important limitation of our comparison of iNPH patients with REF individuals is their different ages, and paravascular clearance has been shown to be impaired with ageing (22). Nevertheless, CSF flow patterns in iNPH patients also differ clearly from those of REF individuals by means of an early and persisting ventricular regurgitation of tracer, as well as by enhancement of periventricular tissue across the ependymal ventricular lining, which was particularly prominent on late scans (Figures 1, 2, 4 and 5). Net tracer flux into brain ventricles may be hypothesized to express a salvage route for CSF flow along the pathway of least resistance and subsequently lead to compromised brain paravascular flow. AQP4 density and its anchoring protein dystrophin 71 have been found to be reduced at astrocytic end feet in iNPH (73). AQP4 loss may cause swelling of end feet and thereby may reduce the distance of inter end feet gaps (74), which may in its turn deteriorate permeability for solutes. The 1-mm image resolution of our MRI exams prevents this direct observation and therefore remains speculative. Furthermore, we note that parenchymal enhancement in iNPH patients was comparable with that of REF individuals (Figure 6) and, in some regions, was more pronounced (Figure 6 and Supplemental Table 4). Increased width of perivascular spaces has been shown to be associated with increasing age (75) and may thus be a determining factor for parenchymal tracer enrichment more than clearance, which has rather been proposed to be dependent on convective forces and AQP4 status in the glymphatic system (7). Our finding is also analogous to those of an MRI study of rats with diabetes mellitus type 2, where perivascular tracer influx was comparable to that in nondiabetes mellitus rats (45). Based on previous data (14), it was suggested that diabetes mellitus type 2 rats have enlarged perivascular spaces owing to increased risk of small vessel disease. This enlargement may therefore reduce resistance to perivascular tracer influx (76).

Moreover, we also found that the correlation between percentage change in tracer enrichment after 24 hours and volume of brain region (gray and white matter and CSF; Table 3) was different between $\mathrm{REF}$ and iNPH individuals. For gray matter of iNPH patients, there were negative correlations between CSF tracer enrichment within the respective region and volume of the specific region, i.e., increased volume of gray matter was associated with lower CSF contrast enrichment. Reduced brain tissue volume in iNPH patients may therefore also have contributed to the observed stronger tracer enrichment in this group at 24 hours. In future studies, CSF tracer clearance assessment with MRI may preferably be compared in patients with minimal cognitive impairment and age-matched healthy controls. This would further reveal the full potential of MRI-based assessment of brain metabolic function to detect neurodegenerative disease in the presymptomatic phase. 
Conclusions. In this study, we have used repeated MRI to demonstrate human brain-wide enrichment of a CSF tracer administered intrathecally. Our observations in human brain add to previous evidence from animal studies, with important distinctions. In humans, CSF tracer distributed centripetally from the surface toward structures in the deep parts of the brain, but over a much longer time course than that reported in animals. Vascular pulsations mediated to CSF seem to play an important role for tracer entry into brain parenchyma. Access of substances to the entire brain extravascular compartment may potentially have large implications for new therapeutic and diagnostic approaches to neurological disease. Delayed tracer clearance from brain parenchyma in patients with iNPH dementia suggests that intrathecal contrast-enhanced MRI could be used to diagnose preclinical neurodegenerative disease.

\section{Methods}

The study was prospective and observational, including consecutive patients with tentative CSF leakage and idiopathic intracranial hypotension and patients with iNPH who underwent MRI before and at several time points following intrathecal lumbar injection of the MRI contrast agent gadobutrol. They were prospectively enrolled from October 2015 to September 2016. Exclusion criteria were history of hypersensitive reactions to contrast agents, history of severe allergy reactions in general, evidence of renal dysfunction, and age $<18$ or $>80$ years as well as pregnancy or breastfeeding for women.

$R E F$ subjects. The individuals with idiopathic intracranial hypotension were referred to the Department of Neurosurgery, Oslo University Hospital - Rikshospitalet, from local neurological departments, based on clinical symptoms and imaging findings indicative of idiopathic intracranial hypotension. They were referred for clinical workup of suspected CSF leakage and underwent MRI with intrathecal gadobutrol, with the primary goal to define site of CSF leakage. These patients were recruited prospectively and consecutively in parallel with iNPH patients. Five of eight REF individuals (REF nos. 1, 2, 4, 5, and 6) have been reported in a previous study (13), though with another method of assessing CSF contrast enrichment and a limited selection of brain regions.

Patients with iNPH dementia. The iNPH patients were referred to the Department of Neurosurgery, Oslo University Hospital - Rikshospitalet, from local neurological departments, based on clinical symptoms and findings indicative of iNPH and imaging findings of ventriculomegaly. Within the Department of Neurosurgery, a clinical assessment was done, and clinical severity was graded based on a previously described NPH grading scale $(77,78)$. Patients were selected for CSF diversion surgery based on a combination of clinical symptoms and findings indicative of iNPH, imaging findings, comorbidity, and results of intracranial pressure monitoring, as previously described $(77,78)$. Four of nine iNPH subjects (iNPH nos. $1,2,3$, and 4) have been reported in a previous study (13), though with another method of assessing CSF contrast enrichment and a limited selection of brain regions.

MRI protocol. We used a 3 Tesla Philips Ingenia MRI scanner (Philips Medical Systems) with equal imaging protocol settings at all time points to acquire sagittal 3D T1-weighted volume scans. The imaging parameters were as follows: repetition time, "shortest" (typically $5.1 \mathrm{~ms}$ ); echo time, "shortest" (typically $2.3 \mathrm{~ms}$ ); Flip angle, 8 degrees; field of view, $256 \times 256 \mathrm{~cm}$; and matrix, $256 \times 256$ pixels (reconstructed 512 $\times 512$ ). We sampled 184 overcontiguous (overlapping) slices with 1-mm thickness, which was automatically reconstructed to 368 slices with $0.5-\mathrm{mm}$ thickness. The total duration of each image acquisition was 6 minutes and 29 seconds. At each time point, imagining stacks were planned using an automated anatomy recognition protocol based on landmark detection in MRI data (SmartExam, Philips Medical Systems) to secure consistency and reproducibility of the MRI slice placement and orientation.

Before gadobutrol administration, we also scanned patients with a sagittal 3D FLAIR volume sequence, where the main imaging parameters were as follows: repetition time $=4,800 \mathrm{~ms}$; echo time, "shortest" (typically $318 \mathrm{~ms}$ ); inversion recovery time, 1,650 ms; field of view, $250 \times 250 \mathrm{~mm}$; and matrix, $250 \times 250$ pixels (reconstructed $512 \times 512$ ). We sampled 184 overcontiguous slices with 1 -mm thickness, which was automatically reconstructed to 365 slices with 0.5 -mm thickness.

Intrathecal administration of gadobutrol. After the precontrast MRI, which was acquired approximately 8 a.m., an interventional neuroradiologist performed x-ray guided lumbar puncture. Correct position of the syringe tip in the SAS was verified by CSF backflow from the puncture needle, and injecting of a small amount (typically $3 \mathrm{ml}$ ) of $270 \mathrm{mg} \mathrm{I} / \mathrm{ml}$ iodixanol (Visipaque, GE Healthcare) confirmed unrestricted distribution of radiopaque contrast agent in the lumbar SAS. Thereafter, intrathecal injection of $0.5 \mathrm{ml}$ of $1.0 \mathrm{mmol} / \mathrm{ml}$ gadobutrol (Gadovist, Bayer) was performed. After needle removal, the study subjects were 
instructed to rotate themselves around the long axis of the body once before transportation back to the MRI suite, while remaining in the supine position. Supplemental Video 3 illustrates the distribution of CSF tracer within the CSF space following intrathecal administration.

Postcontrast MRI acquisitions. Consecutive, identical MRI acquisitions using the previously outlined MRI protocol parameters were performed as soon as possible after intrathecal gadobutrol administration (typically with approximately 10 minutes delay) and performed approximately every 10 minutes during the first hour after contrast agent injection. The study participants were thereafter instructed to remain supine in bed. One pillow under the head was allowed, allowing for 15-degrees head elevation. Repeated, identical image acquisitions were then performed approximately every 2 hours after intrathecal gadobutrol administration until afternoon, at approximately 4 p.m. All transfer of study subjects between the neurosurgical department and the MRI suite, and between the bed and the MRI table, was performed by the hospital staff to allow for the patient to remain in the supine position. The final MRI scanning was performed next morning (approximately 24 hours after contrast agent injection). Patients and controls were allowed to move without any restrictions between the 4 p.m. examination at the end of day 1 and the 24-hour scan next morning.

While the MRI exams, for practical reasons, could not be obtained at identical time points for every study subject, all exams were categorized into the following time intervals: precontrast, 1-2 hours, 2-4 hours, 4-6 hours, 6-9 hours, 24 hours, 48 hours, and after 4 weeks.

Image analysis. The FreeSurfer software (version 6.0) (http://surfer.nmr.mgh.harvard.edu/) was used for segmentation, parcellation, and registration/alignment of the longitudinal data (Supplemental Figures 2 and 3). The segmentation and parcellation acquired from FreeSurfer were used to investigate the increase of $\mathrm{T} 1$ intensity due to CSF tracer. The methods are documented in a review (79). This includes removal of nonbrain tissue using a hybrid watershed/surface deformation procedure (80), automated Talairach transformation, and segmentation of the subcortical white matter and deep gray matter volumetric structures (including hippocampus, amygdala, caudate, putamen, and ventricles) $(81,82)$.

The MR images of each patient were used to create a median template registered to the baseline, a process has been previously described (83). Hence, for each patient, the MR images were registered to the corresponding template using a rigid transformation (83). The registrations were subsequently checked manually by LMV, and no significant errors were visible.

The T2 images (for all iNPH patients except 3) were also used for the segmentation with FreeSurfer. Additionally, the specification of large ventricles was added to the segmentation processes for the NPH patients.

The segmentation of $7 \mathrm{iNPH}$ patients was edited due to segmentation errors. These editions were due to severe segmentation errors, such as mislabeling of ventricle volume as white matter and the inclusion of dura as part of the parenchyma; other minor errors were not considered. These corrections were automatic based on the patient T2 image, except in the case of 1 iNPH patient, which was done manually.

Parameters derived from gadobutrol enhancement. For each segmented area, we computed the median T1 signal unit for each time point. Further, we divided the median signal unit against the signal unit of a REF ROI placed within the posterior part of the superior sagittal sinus in axially reconstructed images from the same T1 volume scan (Supplemental Figure 4). We referred to the result of this as normalized T1 signal units; this process corrects for any baseline changes of image gray scale due to image scaling. Previous observations indicate no measurable enhancement of contrast agent at MRI in the REF location after intrathecal injection of gadobutrol at this concentration (13).

CSF contrast enrichment and volume of brain region. For REF and iNPH subjects, we determined Pearson correlation coefficients by comparing the percentage change in signal unit ratio after 24 hours and the volume of the specific brain region (white and gray matter and CSF of lateral ventricles). Volumes were computed by summation of the number of voxels of every subregion in the segmentation and multiplication of the result by the voxel size of $1.0 \mathrm{~mm}^{3}$.

Statistics. Categorical data are shown as the number of observations (percentage) and continuous data are shown as mean (SD) or mean (SEM), as appropriate. We estimated from the image analysis the mean response for each patient at 0 (precontrast), 1.5-2.0, 2-4, 4-6, 6-9, 24, and 48 hours and at 4 weeks follow-up. The repeated measurements were assessed with linear mixed models using a random intercept, robust SEM, and maximum likelihood estimation. Due to few observations, the results at 48 hours were omitted from the statistical model. Using a linear combination from the statistical model, we tested the difference between the REF and iNPH groups at the different points of follow-up. 
For the statistical analysis, we used SPSS version 22 (IBM Corporation) or Stata/SE 15.0 (StataCrop LLX). Statistical significance was accepted at the 0.05 level (2-tailed).

Study approval. The Regional Committee for Medical and Health Research Ethics of Health, South East Region, Norway (2015/96); the Institutional Review Board of Oslo University Hospital (2015/1868); and the National Medicines Agency, Norway (15/04932-7) approved the study. The study participants were included after written and oral informed consent.

\section{Author contributions}

GR, LMV, KAM, and PKE conceptualized and designed the study. GR, LMV, AMD, AHP, SASV, KEE, KAM, and PKE provided investigation and formal analysis. GR, KAM, and PKE supervised and administrated the study and wrote the original draft. GR, LMV, AMD, AHP, SASV, KEE, KAM, and PKE wrote, reviewed, and edited the manuscript. All authors (GR, LMV, AMD, AHP, SASV, KEE, KAM, and PKE) approved the final manuscript.

\section{Acknowledgments}

We thank Øivind Gjertsen, Bård Nedregaard, and Ruth Sletteberg from the Department of Radiology, Oslo University Hospital - Rikshospitalet, who performed the intrathecal gadobutrol injections in all study subjects. We also sincerely thank the Intervention Centre and Department of Neurosurgery at Oslo University Hospital - Rikshospitalet for providing valuable support with MR scanning and care for all study subjects throughout the study. Finally, we sincerely thank the Nursing Staff and Hydrocephalus Outward Clinic, Department of Neurosurgery Oslo University Hospital - Rikshospitalet, for care of all study subjects throughout the study.

Address correspondence to: Per K. Eide, Department of Neurosurgery, Oslo University Hospital — Rikshospitalet, Pb 4950 Nydalen, N-0424 Oslo, Norway. Phone: 47.2307.4321; Email: p.k.eide@medisin.uio. no or peide@ous-hf.no.

1. Wolak DJ, Thorne RG. Diffusion of macromolecules in the brain: implications for drug delivery. Mol Pharm. 2013;10(5):1492-1504.

2. Schlageter KE, Molnar P, Lapin GD, Groothuis DR. Microvessel organization and structure in experimental brain tumors: microvessel populations with distinctive structural and functional properties. Microvasc Res. 1999;58(3):312-328.

3. Pangalos MN, Schechter LE, Hurko O. Drug development for CNS disorders: strategies for balancing risk and reducing attrition. Nat Rev Drug Discov. 2007;6(7):521-532.

4. Finkel RS, et al. Treatment of infantile-onset spinal muscular atrophy with nusinersen: a phase 2, open-label, dose-escalation study. Lancet. 2016;388(10063):3017-3026.

5. Pizzo ME, et al. Intrathecal antibody distribution in the rat brain: surface diffusion, perivascular transport and osmotic enhancement of delivery. J Physiol (Lond). 2018;596(3):445-475.

6. Rennels ML, Gregory TF, Blaumanis OR, Fujimoto K, Grady PA. Evidence for a 'paravascular' fluid circulation in the mammalian central nervous system, provided by the rapid distribution of tracer protein throughout the brain from the subarachnoid space. Brain Res. 1985;326(1):47-63.

7. Iliff JJ, et al. A paravascular pathway facilitates CSF flow through the brain parenchyma and the clearance of interstitial solutes, including amyloid $\beta$. Sci Transl Med. 2012;4(147):147ra111.

8. Bedussi B, Almasian M, de Vos J, VanBavel E, Bakker EN. Paravascular spaces at the brain surface: Low resistance pathways for cerebrospinal fluid flow. J Cereb Blood Flow Metab. 2018;38(4):719-726.

9. Smith AJ, Yao X, Dix JA, Jin BJ, Verkman AS. Test of the 'glymphatic' hypothesis demonstrates diffusive and aquaporin-4-independent solute transport in rodent brain parenchyma. Elife. 2017;6:e27679.

10. Dobson H, et al. The perivascular pathways for influx of cerebrospinal fluid are most efficient in the midbrain. Clin Sci. 2017;131(22):2745-2752.

11. Lam MA, Hemley SJ, Najafi E, Vella NGF, Bilston LE, Stoodley MA. The ultrastructure of spinal cord perivascular spaces: Implications for the circulation of cerebrospinal fluid. Sci Rep. 2017;7(1):12924.

12. Eide PK, Ringstad G. MRI with intrathecal MRI gadolinium contrast medium administration: a possible method to assess glymphatic function in human brain. Acta Radiol Open. 2015;4(11):2058460115609635.

13. Ringstad G, Vatnehol SAS, Eide PK. Glymphatic MRI in idiopathic normal pressure hydrocephalus. Brain. 2017;140(10):2691-2705.

14. Iliff JJ, et al. Brain-wide pathway for waste clearance captured by contrast-enhanced MRI. J Clin Invest. 2013;123(3):1299-1309.

15. Carare RO, et al. Solutes, but not cells, drain from the brain parenchyma along basement membranes of capillaries and arteries: significance for cerebral amyloid angiopathy and neuroimmunology. Neuropathol Appl Neurobiol. 2008;34(2):131-144.

16. Morris AW, et al. Vascular basement membranes as pathways for the passage of fluid into and out of the brain. Acta Neuropathol. 2016;131(5):725-736.

17. Syková E, Nicholson C. Diffusion in brain extracellular space. Physiol Rev. 2008;88(4):1277-1340

18. Iliff JJ, et al. Cerebral arterial pulsation drives paravascular CSF-interstitial fluid exchange in the murine brain. $J$ Neurosci. 2013;33(46):18190-18199. 
19. Zeppenfeld DM, et al. Association of perivascular localization of aquaporin-4 with cognition and Alzheimer disease in aging brains. JAMA Neurol. 2017;74(1):91-99.

20. Xia M, Yang L, Sun G, Qi S, Li B. Mechanism of depression as a risk factor in the development of Alzheimer's disease: the function of AQP4 and the glymphatic system. Psychopharmacology (Berl). 2017;234(3):365-379.

21. Iliff JJ, et al. Impairment of glymphatic pathway function promotes tau pathology after traumatic brain injury. $J$ Neurosci. 2014;34(49):16180-16193.

22. Kress BT, et al. Impairment of paravascular clearance pathways in the aging brain. Ann Neurol. 2014;76(6):845-861.

23. Xie L, et al. Sleep drives metabolite clearance from the adult brain. Science. 2013;342(6156):373-377.

24. Ju YE, et al. Obstructive sleep apnea decreases central nervous system-derived proteins in the cerebrospinal fluid. Ann Neurol. 2016;80(1):154-159

25. He XF, et al. Voluntary exercise promotes glymphatic clearance of amyloid beta and reduces the activation of astrocytes and microglia in aged mice. Front Mol Neurosci. 2017;10:144.

26. Jin BJ, Smith AJ, Verkman AS. Spatial model of convective solute transport in brain extracellular space does not support a "glymphatic" mechanism. J Gen Physiol. 2016;148(6):489-501.

27. Holter KE, et al. Interstitial solute transport in 3D reconstructed neuropil occurs by diffusion rather than bulk flow. Proc Natl Acad Sci USA. 2017;114(37):9894-9899.

28. Mestre H, et al. Aquaporin-4 dependent glymphatic solute transport in rodent brain. bioRxiv. https://www.biorxiv.org/content/ early/2017/11/09/216499. Published November 9, 2017. Accessed June 18, 2018

29. Thorne RG, Hrabetová S, Nicholson C. Diffusion of epidermal growth factor in rat brain extracellular space measured by integrative optical imaging. J Neurophysiol. 2004;92(6):3471-3481.

30. Nicholson C, Tao L. Hindered diffusion of high molecular weight compounds in brain extracellular microenvironment measured with integrative optical imaging. Biophys J. 1993;65(6):2277-2290.

31. Thorne RG, Nicholson C. In vivo diffusion analysis with quantum dots and dextrans predicts the width of brain extracellular space. Proc Natl Acad Sci USA. 2006;103(14):5567-5572.

32. WOOLLAM DH, MILLEN JW. The perivascular spaces of the mammalian central nervous system and their relation to the perineuronal and subarachnoid spaces. J Anat. 1955;89(2):193-200.

33. MILLEN JW, WOOLLAM DH. On the nature of the pia mater. Brain. 1961;84:514-520.

34. Hutchings M, Weller RO. Anatomical relationships of the pia mater to cerebral blood vessels in man. J Neurosurg. 1986;65(3):316-325

35. Zhang ET, Inman CB, Weller RO. Interrelationships of the pia mater and the perivascular (Virchow-Robin) spaces in the human cerebrum. J Anat. 1990;170:111-123.

36. Pollock H, Hutchings M, Weller RO, Zhang ET. Perivascular spaces in the basal ganglia of the human brain: their relationship to lacunes. J Anat. 1997;191(Pt 3):337-346.

37. Engelhardt B, Vajkoczy P, Weller RO. The movers and shapers in immune privilege of the CNS. Nat Immunol. 2017;18(2):123-131.

38. Asgari M, de Zélicourt D, Kurtcuoglu V. Glymphatic solute transport does not require bulk flow. Sci Rep. $2016 ; 6: 38635$.

39. Bedussi B, et al. Paravascular channels, cisterns, and the subarachnoid space in the rat brain: A single compartment with preferential pathways. J Cereb Blood Flow Metab. 2017;37(4):1374-1385.

40. Abbott NJ. Evidence for bulk flow of brain interstitial fluid: significance for physiology and pathology. Neurochem Int. 2004;45(4):545-552.

41. Rosenberg GA, Kyner WT, Estrada E. Bulk flow of brain interstitial fluid under normal and hyperosmolar conditions. Am $J$ Physiol. 1980;238(1):F42-F49.

42. Hannocks MJ, et al. Molecular characterization of perivascular drainage pathways in the murine brain. J Cereb Blood Flow Metab. 2018;38(4):669-686.

43. Cserr HF. Physiology of the choroid plexus. Physiol Rev. 1971;51(2):273-311.

44. Cserr HF, Cooper DN, Suri PK, Patlak CS. Efflux of radiolabeled polyethylene glycols and albumin from rat brain. Am J Physiol. 1981;240(4):F319-F328.

45. Jiang Q, et al. Impairment of the glymphatic system after diabetes. J Cereb Blood Flow Metab. 2017;37(4):1326-1337.

46. Bakker EN, et al. Lymphatic clearance of the brain: perivascular, paravascular and significance for neurodegenerative diseases. Cell Mol Neurobiol. 2016;36(2):181-194.

47. Calias $\mathrm{P}$, et al. CNS penetration of intrathecal-lumbar idursulfase in the monkey, dog and mouse: implications for neurological outcomes of lysosomal storage disorder. PLOS ONE. 2012;7(1):e30341.

48. Papisov MI, Belov VV, Gannon KS. Physiology of the intrathecal bolus: the leptomeningeal route for macromolecule and particle delivery to CNS. Mol Pharm. 2013;10(5):1522-1532.

49. Papisov MI, et al. Investigation of intrathecal transport of NPT002, a prospective therapeutic based on phage M13, in nonhuman primates. Drug Deliv Transl Res. 2012;2(3):210-221

50. Brinker T, Stopa E, Morrison J, Klinge P. A new look at cerebrospinal fluid circulation. Fluids Barriers CNS. $2014 ; 11: 10$.

51. Aspelund A, et al. A dural lymphatic vascular system that drains brain interstitial fluid and macromolecules. $J$ Exp Med. 2015;212(7):991-999.

52. Louveau A, et al. Structural and functional features of central nervous system lymphatic vessels. Nature. 2015;523(7560):337-341.

53. Eide PK, Vatnehol SAS, Emblem KE, Ringstad G. Magnetic resonance imaging provides evidence of glymphatic drainage from human brain to cervical lymph nodes. Sci Rep. 2018;8(1):7194.

54. Ma Q, Ineichen BV, Detmar M, Proulx ST. Outflow of cerebrospinal fluid is predominantly through lymphatic vessels and is reduced in aged mice. Nat Commun. 2017;8(1):1434.

55. Pardridge WM. The blood-brain barrier: bottleneck in brain drug development. NeuroRx. 2005;2(1):3-14.

56. Bielekova B, et al. Intrathecal effects of daclizumab treatment of multiple sclerosis. Neurology. 2011;77(21):1877-1886.

57. Bottros MM, Christo PJ. Current perspectives on intrathecal drug delivery. J Pain Res. 2014;7:615-626.

58. Robert R, Wark KL. Engineered antibody approaches for Alzheimer's disease immunotherapy. Arch Biochem Biophys. 2012;526(2):132-138. 
59. Kilsdonk ID, et al. Increased cortical grey matter lesion detection in multiple sclerosis with $7 \mathrm{~T}$ MRI: a post-mortem verification study. Brain. 2016;139(Pt 5):1472-1481.

60. Cuddapah VA, Robel S, Watkins S, Sontheimer H. A neurocentric perspective on glioma invasion. Nat Rev Neurosci. 2014;15(7):455-465.

61. Kanda T, Ishii K, Kawaguchi H, Kitajima K, Takenaka D. High signal intensity in the dentate nucleus and globus pallidus on unenhanced T1-weighted MR images: relationship with increasing cumulative dose of a gadolinium-based contrast material. Radiology. 2014;270(3):834-841.

62. Kanda T, Nakai Y, Hagiwara A, Oba H, Toyoda K, Furui S. Distribution and chemical forms of gadolinium in the brain: a review. Br J Radiol. 2017;90(1079):20170115.

63. Jost G, Frenzel T, Lohrke J, Lenhard DC, Naganawa S, Pietsch H. Penetration and distribution of gadolinium-based contrast agents into the cerebrospinal fluid in healthy rats: a potential pathway of entry into the brain tissue. Eur Radiol. 2017;27(7):2877-2885.

64. Radbruch A, et al. Pediatric brain: no increased signal intensity in the dentate nucleus on unenhanced T1-weighted MR images after consecutive exposure to a macrocyclic gadolinium-based contrast agent. Radiology. 2017;283(3):828-836.

65. Radbruch A, et al. No signal intensity increase in the dentate nucleus on unenhanced T1-weighted MR images after more than 20 serial injections of macrocyclic gadolinium-based contrast agents. Radiology. 2017;282(3):699-707.

66. Yang L, et al. Evaluating glymphatic pathway function utilizing clinically relevant intrathecal infusion of CSF tracer. $J$ Transl Med. 2013;11:107.

67. Lowe J, Mirra SS, Hyman BT, Dickson DW. Chapter 14: Ageing and Dementia. In: Love S, Louis D, Ellison DW, eds. Greenfield's Neuropathology. Boca Raton, FL, USA: CRC Press; 2008:1031-1152.

69. Eide PK, Ringstad G. Delayed clearance of cerebrospinal fluid tracer from entorhinal cortex in idiopathic normal pressure hydrocephalus: A glymphatic magnetic resonance imaging study [published online ahead of print February 27, 2018]. J Cereb Blood Flow Metab. https://doi.org/10.1177/0271678X18760974.

68. Golomb J, et al. Alzheimer's disease comorbidity in normal pressure hydrocephalus: prevalence and shunt response. J Neurol Neurosurg Psychiatry. 2000;68(6):778-781.

70. Pennanen C, et al. Hippocampus and entorhinal cortex in mild cognitive impairment and early AD. Neurobiol Aging. 2004;25(3):303-310.

71. Israelsson H, et al. Vascular risk factors in INPH: A prospective case-control study (the INPH-CRasH study). Neurology. 2017;88(6):577-585.

72. de Calignon A, et al. Propagation of tau pathology in a model of early Alzheimer's disease. Neuron. 2012;73(4):685-697.

73. Eide PK, Hansson HA. Astrogliosis impaired aquaporin-4 dystrophin systems in idiopathic normal pressure hydrocephalus [published online ahead of print June 19, 2018]. Neuropathol Appl Neurobiol. https://doi.org/10.1111/nan.12420.

74. Amiry-Moghaddam M, et al. An alpha-syntrophin-dependent pool of AQP4 in astroglial end-feet confers bidirectional water flow between blood and brain. Proc Natl Acad Sci USA. 2003;100(4):2106-2111.

75. Heier LA, Bauer CJ, Schwartz L, Zimmerman RD, Morgello S, Deck MD. Large Virchow-Robin spaces: MR-clinical correlation. AJNR Am J Neuroradiol. 1989;10(5):929-936.

76. Mestre H, Kostrikov S, Mehta RI, Nedergaard M. Perivascular spaces, glymphatic dysfunction, and small vessel disease. Clin Sci. 2017;131(17):2257-2274.

77. Eide PK, Sorteberg W. Diagnostic intracranial pressure monitoring and surgical management in idiopathic normal pressure hydrocephalus: a 6-year review of 214 patients. Neurosurgery. 2010;66(1):80-91.

78. Eide PK, Sorteberg W. Outcome of surgery for idiopathic normal pressure hydrocephalus: role of preoperative static and pulsatile intracranial pressure. World Neurosurg. 2016;86:186-193.e1.

79. Fischl B. FreeSurfer. Neuroimage. 2012;62(2):774-781

80. Ségonne F, et al. A hybrid approach to the skull stripping problem in MRI. Neuroimage. 2004;22(3):1060-1075.

81. Fischl B, et al. Whole brain segmentation: automated labeling of neuroanatomical structures in the human brain. Neuron. 2002;33(3):341-355.

82. Fischl B, et al. Sequence-independent segmentation of magnetic resonance images. Neuroimage. 2004;23 Suppl 1:S69-S84.

83. Reuter M, Schmansky NJ, Rosas HD, Fischl B. Within-subject template estimation for unbiased longitudinal image analysis Neuroimage. 2012;61(4):1402-1418. 